\title{
Acessos cirúrgicos combinados para tratamento de fraturas em assoalho orbital e lâmina papirácea: relato de caso
}

\author{
Combined surgical accesses for treatment of fractures in the orbital floor and \\ lamina papyracea: case report
}

Verônica Cristine Rodrigues Costa ${ }^{1}$, Rosalvo Streit Junior ${ }^{1}$, Juliana da Luz Araújo ${ }^{2}$, Elisa Vasconcelos de Queiroz $^{3}$, Felipe Carneiro Krier ${ }^{2}$, Diderot Rodrigues Parreira ${ }^{4}$, Rodrigo Tamietti Durães ${ }^{5}$

\begin{abstract}
RESUMO
A região orbitária é bastante suscetível a traumas, visto que apresenta uma posição exposta, além de ser composta por ossos frágeis. Quando indicada, é preciso intervenção cirúrgica para preservar a função visual e harmonia facial do paciente. Os acessos transconjuntival e transcaruncular são descritos na literatura como formas seguras, rápidas, funcionais e esteticamente benéficas para a abordagem ao assoalho orbitário e lâmina papirácea. Sobre os materiais biocompatíveis utilizados, a placa de titânio é inabsorvível e permite fixação interna rígida, moldada às curvaturas naturais dos ossos, com baixo risco de infecção. As folhas de polietileno poroso são polímeros inertes e não absorvíveis que facilitam o crescimento de tecido e reduzem as chances de rejeição. Nesse relato de caso, evidencia-se paciente masculino, 28 anos, vítima de traumatismo facial após prática esportiva que apresentou fratura de assoalho e parede medial orbitária direita, com indicação de reconstrução orbitária com acessos transconjuntival e transcaruncular para fixação de placa de titânio em assoalho da órbita direita e de folhas de polietileno poroso em lâmina papirácea à direita, respectivamente.
\end{abstract}

Palavras-chave: Fraturas Orbitárias. Acesso Transconjuntival. Acesso Transcaruncular.

\begin{abstract}
The orbital region is quite susceptible to trauma, since it has an exposed position, besides being composed of fragile bones. When indicated, surgical intervention is required to preserve the patient's visual function and facial harmony. Transconjunctival and transcaruncular accesses are described in the literature as safe, fast, functional and with esthetic benefits for the approach to the orbital floor and papyraceous lamina. Among the biocompatible materials used, the titanium plate does not undergo resorption and allows rigid internal fixation, being shaped to the natural curvatures of the bones, with low risk of infection. Porous polyethylene sheets are inert and nonabsorbable polymers that facilitate tissue growth and
\end{abstract}

1. Acadêmicos de medicina da Universidade Católica de Brasília

2. Médicos graduados pela Universidade Católica de Brasília

3. Acadêmica de medicina do Centro Universitário de Brasília

4. Médico otorrinolaringologista e cirurgião crânio-maxilofacial. Docente do curso de medicina da Universidade Católica de Brasília

5. Médico oftalmologista
CORRESPONDÊNCIA: Verônica Cristine Rodrigues Costa Universidade Católica de Brasília QS 07, Lote 01, EPCT CEP 71966-700 - Brasília/DF - Brasil veronicacristine0@gmail.com

Recebido em 19/09/2018 Aprovado em 30/01/2019 
reduce the chances of rejection. In this case report, a 28-year-old male patient, a victim of facial trauma after sports practice presented a fracture of the floor and the right orbital medial wall, and orbital reconstruction was indicated with transconjunctival and transcaruncular accesses for fixation of titanium plate in the floor of the right orbit and porous polyethylene sheets in papyracea leaf on the right, respectively.

Keywords: Orbital Fractures. Transconjunctival Access. Transcaruncular Access.

\section{INTRODUÇÃO}

A região orbitária é bastante suscetível a fraturas porque apresenta uma posição exposta, além de ser composta por ossos frágeis. ${ }^{1}$ Dados epidemiológicos internacionais apontam o assoaIho orbitário como quarto lugar entre os traumas craniofaciais, precedido pelo osso nasal, mandibular e malar-maxilar, ${ }^{2}$ enquanto os dados nacionais apresentam o complexo zigomático-orbital como a região facial mais fraturada. ${ }^{3}$

Nessas situações, o diagnóstico é feito com a história clínica e tomografia computadorizada (TC) de face e, quando indicada, é necessária reconstrução cirúrgica para restaurar a estrutura anatômica da órbita, preservar a função visual e a harmonia da face. ${ }^{1}$ Nesse relato de caso serão abordados os acessos transconjuntival e transcaruncular para exposição do assoalho e parede medial orbitária, respectivamente. Cirurgias nessa região são consideradas desafiadoras, visto que possui delicada proximidade com estruturas importantes, como o aparelho lacrimal e tendão cantal medial, além do formato tridimensional das paredes orbitárias, já que são curvas. ${ }^{4}$

\section{RELATO DE CASO}

Paciente masculino, previamente hígido, 28 anos, solteiro, procurou atendimento médico, em setembro de 2016, após traumatismo facial decorrente de práticas de arte marcial (muay thai) há 6 dias. Ao exame físico, apresentava enoftalmia do olho direito e hematoma com desvio de pirâmide nasal. Em TC de face com reconstrução em 3D (Figura 1), evidenciou-se fratura de base de osso nasal à direita com mínimo desvio medial, de assoalho de órbita no teto de seio maxilar direito com pequena herniação de gordura e de parede medial orbitária em lâmina papirácea do osso etmoide, sendo indicada reconstrução orbitária.
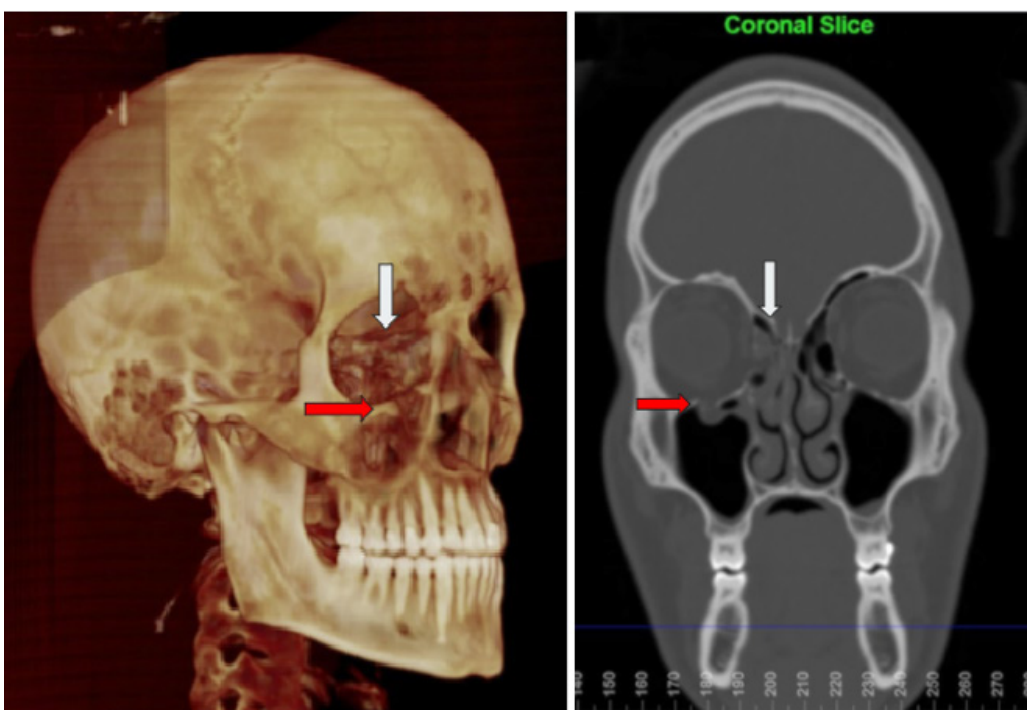

Figura 1: Tomografia computadorizada de face pré-operatória com presença de fratura de parede medial orbitária (seta branca) e em assoalho da órbita (seta vermelha). 
Assim, realizou-se cirurgia com acessos transconjuntival e transcaruncular em hemiface direita (Figura 2) com dissecação de planos anatômicos até a completa visualização do assoalho da órbita e da lâmina papirácea. A fratura do rebordo orbitário inferior foi reduzida e, em seguida, uma placa de titânio foi fixada com parafusos do mesmo material.
$\mathrm{Na}$ parede medial orbitária, foram posicionadas folhas de polietileno poroso Medpor ${ }^{\circledR}$. Posteriormente, foi realizado teste de mobilidade da musculatura extrínseca com pinçamento para verificar se houve encarceramento muscular. $\mathrm{O}$ procedimento ocorreu sem intercorrências e o paciente evoluiu adequadamente no pós-operatório (Figura 3).

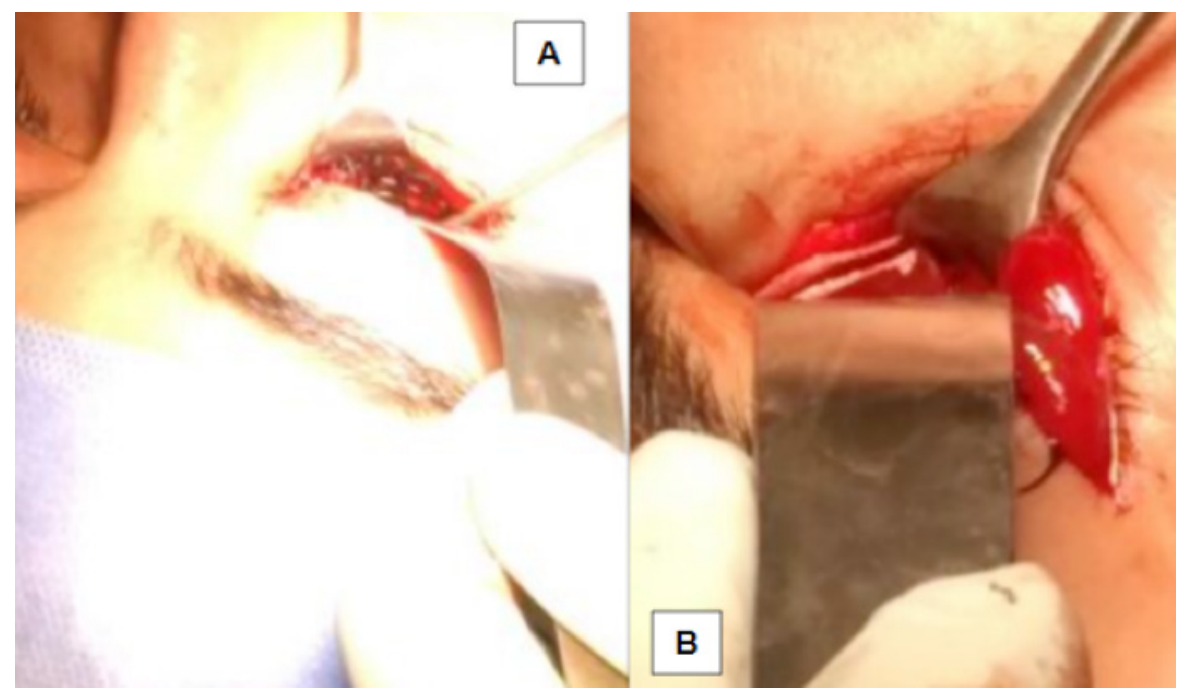

Figura 2: A) Acesso transconjuntival para fixação de placa de titânio. B) Inserção de folha de Medpor ${ }^{\circledR}$ por acesso transcaruncular.

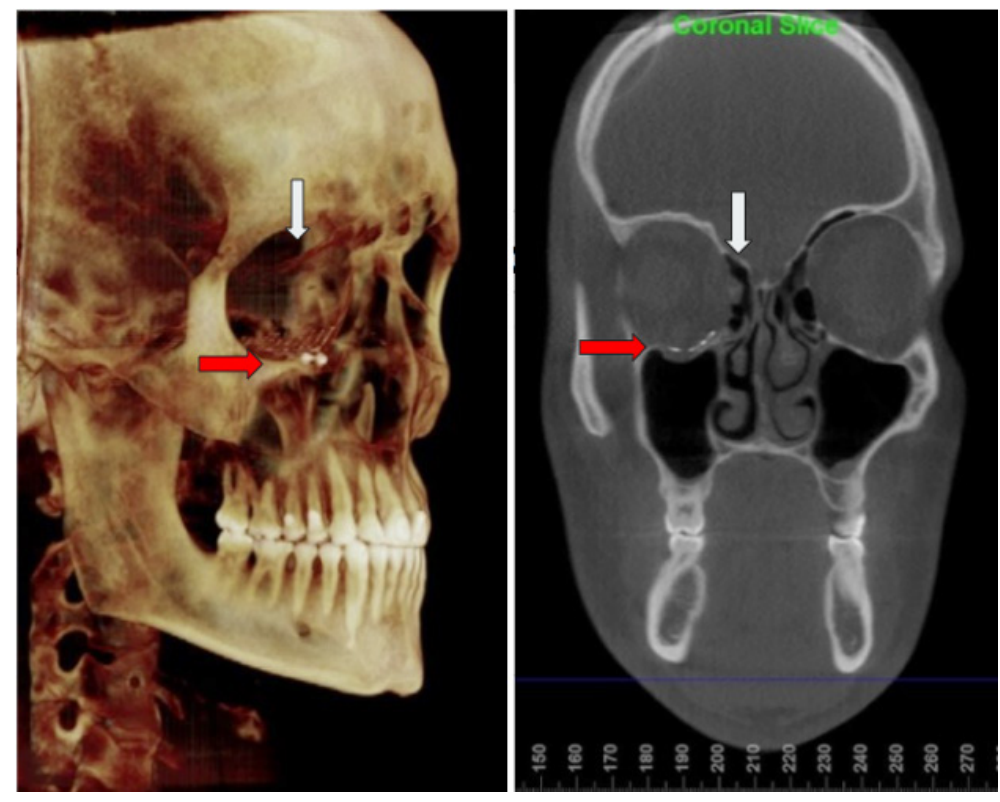

Figura 3: Tomografia computadorizada de face pós-operatória, evidenciando presença da placa de titânio (seta vermelha) e preservação anatômica da parede orbitária. 


\section{DISCUSSÃO}

Em relação às fraturas de órbita, estudos epidemiológicos apontam as causas mais comuns, cuja ordem de incidência pode variar de acordo com as características da região analisada. Nos Estados Unidos, as principais são agressões físicas (24,9\%), acidentes de trânsito $(21,7 \%)$, lesões em atividades cotidianas $(15,3 \%)$ e prática de esportes $(15,3 \%) .{ }^{1}$ No Brasil, as circunstâncias mais comuns envolvem acidentes de trânsito (58\%), agressão física (15\%), quedas $(12 \%)$ e esportes $(7 \%) .^{5}$

Esse estudo retrospectivo realizado no Brasil analisou, estatisticamente, em um período de sete anos, 141 pacientes com fratura do complexo órbito-zigomático que necessitaram de tratamento cirúrgico. Observou-se que esse tipo de fratura predomina no sexo masculino ( $86,5 \%)$ e na faixa etária entre 21 e 30 anos. Os principais sinais e sintomas clínicos são equimoses/hematomas $(46,2 \%)$, deformidades ósseas palpáveis (31,9\%), depressão malar $(28,6 \%)$, limitação do movimento da mandíbula $(14,3 \%)$, diplopia $(13,2 \%)$ e enoftalmia $(12,1 \%)$. Do total, 137 pacientes apresentaram fratura unilateral, enquanto sete apresentaram bilateral. ${ }^{5}$

Desse modo, de acordo com estudos, nos EUA, o trauma por prática de esportes ocupa a quarta posição entre as principais causas. ${ }^{1} \mathrm{O}$ mesmo ocorre no Brasil, com predomínio de homens entre
21 e 30 anos, faixa etária apresentada pelo paciente, que manifestou, ao exame clínico, achados como enoftalmia, hematoma e deformidade óssea palpável. ${ }^{5}$ Estudo retrospectivo com 483 fraturas de órbita em Singapura analisou padrões de traumas nas 65 dessas relacionadas ao esporte, sendo observado maior incidência do complexo zigomático-maxilar $(36,9 \%)$, fraturas isoladas de uma parede $(29,2 \%)$, sendo mais comum o assoalho da órbita e fraturas naso-orbito-etmoidais $(10,8 \%){ }^{6}$

As indicações gerais para a intervenção cirúrgica incluem sinais e sintomas, como diplopia, enoftalmia, exoftalmia, mobilidade ocular prejudicada, evidência tomográfica de fraturas extensas. ${ }^{1,4}$ Os acessos transconjuntival e transcaruncular (Figura 4), indicados para reparo de fraturas orbitárias, são apontados como bons métodos em relação aos transcutâneos. O paciente é submetido a anestesia geral e permanece em posição supina. Um anestésico local com epinefrina é aplicado na conjuntiva para fins hemostáticos. Inicialmente, verifica-se a motilidade passiva do globo ocular por meio do teste de ducção forçada. Uma sutura de tração é colocada na parte inferior da pálpebra, sendo identificada a borda inferior do tarso e, assim, realiza-se a incisão transconjuntival para exposição do assoalho da órbita. ${ }^{7}$ Para proteger a córnea, recomenda-se que seja coberta pelo retalho conjuntival por meio de tração superior e feita sutura na extremidade superior. ${ }^{8}$
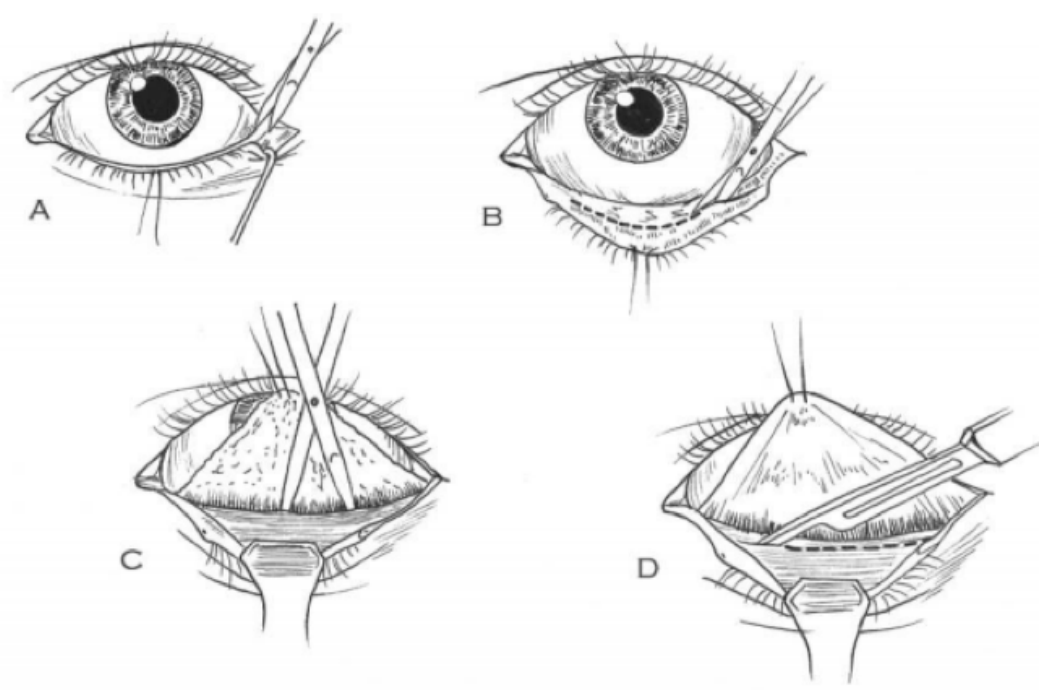

Figura 4: Combinação dos acessos transconjuntival e transcaruncular. A) Incisão na fissura palpebral com extensão caruncular. B) Com a pálpebra evertida, conjuntiva é incisada. C) Proteção da córnea com retalho conjuntival. D) Incisão no periósteo. 7,8 
$\mathrm{Na}$ abordagem transcaruncular (Figura 5), a incisão é cuidadosamente realizada entre a carúncula lacrimal e a plica semilunaris, realizando-se a dissecação dos tecidos moles até o periósteo da parede orbitária medial. Nesse processo, é preciso ter cuidado para não danificar o músculo reto medial e o saco lacrimal. Vale ressaltar que, nessa abordagem, é obrigatória a utilização de um protetor para a córnea de coloração transparente, para evitar lesões, permitir a detecção precoce de midríase e evitar neuropatia óptica em torno do anel óptico. Apesar de oferecer acesso direto e previsível à região orbitária medial e evitar lesões no aparelho lacrimal, observa-se que a técnica isolada possui limitado campo cirúrgico, o que dificulta grandes implantes. ${ }^{9}$

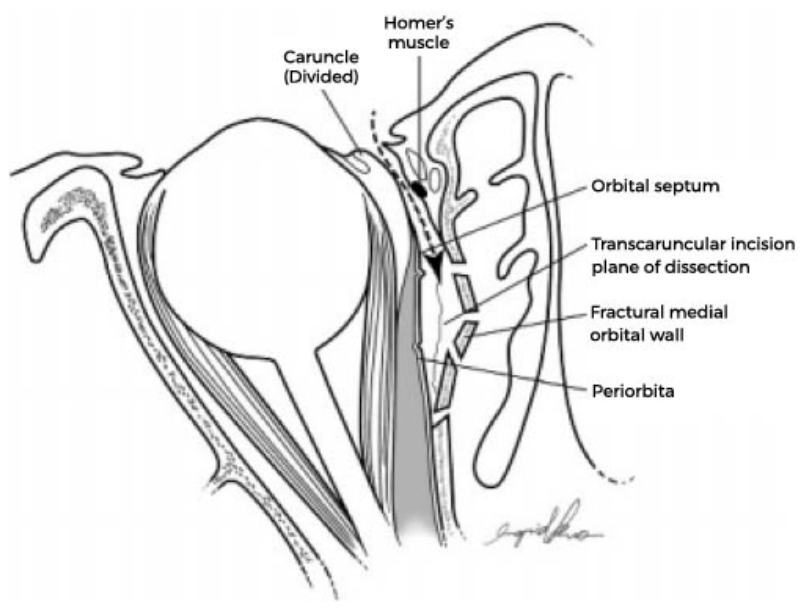

Figura 5: Inserção de folhas de Medpor ${ }^{\circledR}$ por acesso transcaruncular (seta pontilhada). ${ }^{7}$

A combinação dos acessos transconjuntival e transcaruncular possibilita uma melhor visualização cirúrgica para grandes fraturas orbitárias, já que a parede orbitária medial é acessada ao estender a incisão transconjuntival através da carúncula lacrimal, o que oferece melhor campo operacional para redução da fratura, posicionamento dos tecidos moles herniados e dos materiais para restauração. Além disso, oferece outras vantagens, como ausência de cicatrizes cutâneas, menores taxas de complicações cirúrgicas e traumas iatrogênicos. ${ }^{4,7}$
Um estudo prospectivo realizado com 40 pacientes, com igual distribuição entre grupos, comparou os acessos transconjuntival com cantotomia lateral inferior, quando necessário, e o subciliar. A duração média entre as incisões até a exposição da fratura foi de 10-16 minutos na abordagem subciliar e 11-18 minutos na transconjuntival, o que não foi significativo. A dor pós-operatória na primeira semana foi tolerável em $90 \%$ dos acessos subciliares e em $85 \%$ dos transconjuntivais, porém, apresentaram algumas restrições como rir e lavar o rosto. A dor intolerável ocorreu em 10\% dos subciliares e $15 \%$ dos transconjuntivais, com diferença estatisticamente não significativa. Em ambos, a dor cedeu após uma ou duas semanas. ${ }^{10}$

No acesso subciliar, $10 \%$ apresentaram ectrópio e $15 \%$, esclera aparente. O transconjuntival não apresentou essas complicações, mas o resultado não foi considerável. Na abordagem transconjuntival, $20 \%$ dos pacientes submetidos apresentaram entrópio, que foi transitório. Nenhum dos pacientes submetidos ao subciliar passaram por tal complicação, com diferença estatisticamente significante. Ambos os grupos apresentaram edema periorbital pós-operatório que diminuiu completamente em todos os casos na segunda semana pós-operatória. $O$ estudo não observou infecção, hematoma ou qualquer outra complicação tardia. Assim, o acesso transconjuntival foi apresentado como uma ferramenta para evitar ectrópio e esclera aparente, especialmente em fraturas mais severas. ${ }^{10}$

Sobre os materiais utilizados, as placas de titânio fixadas no assoalho orbitário são materiais biocompatíveis capazes de manter e posicionar a região fraturada. Elas permitem uma fixação interna rígida, de maneira tridimensional, e ainda podem ser moldadas conforme as curvaturas naturais dos ossos envolvidos. Com isso, assegura-se uma correção anatômica precisa, com resultados favoráveis a longo prazo, baixos riscos de complicações e mínima reação de corpo estranho. Como não estão sujeitas a reabsorção, são capazes de compensar o volume orbitário fraturado. São implantes ideais para cobrir grandes defeitos anatômicos. No entanto, ainda possuem alto custo, são necessárias incisões mais amplas e, se não cortadas corretamente, pode haver bordas irregulares que interferem em tecidos moles. ${ }^{9}$ 
Em relação às folhas de Medpor $^{\circledR}$ posicionadas na lâmina papirácea, trata-se de um polímero inerte e não absorvível, formado por uma rede de poros abertos e interligados, que facilitam o crescimento do tecido adjacente e reduzem as chances de rejeição ao corpo estranho. ${ }^{9}$ É um material estável, biocompatível, facilmente contornável, de dureza moderada e associado a poucas complicações. Entretanto, deve-se observar o risco de adesão aos músculos extraoculares e possui alto custo.?

\section{CONCLUSÃO}

Ao considerar a prevalência e as diferentes etiologias que podem ocasionar fraturas de órbitas, observa-se a importância da escolha do acesso cirúrgico à órbita e de materiais biocompatíveis que proporcionem melhor qualidade de vida ao paciente. Dessa forma, as técnicas transconjuntival e transcaruncular são descritas na literatura como formas seguras, rápidas, funcionais e esteticamente benéficas o que, consequentemente, propiciam resultados favoráveis para a abordagem ao assoalho orbitário e lâmina papirácea.

\section{REFERÊNCIAS}

1. Gosau M, Schöneich M, Draenert FG, Ettl T, Driemel $\mathrm{O}$, Reichert TE. Retrospective analysis of orbital floor fractures - complications, outcome, and review of literature. Clin Oral Investig. 2011;15:305-13.
2. Streubel SO, Mirsky DM. Craniomaxillofacial trauma. Facial Plast Surg Clin N Am. 2016; 24: 605-17.

3. Porto DE, Carreira PFS, Cavalcante JR. Análise do tempo médio de internação em pacientes com fraturas faciais em hospitais de urgência e emergência da Paraíba - PB. Rev Cir Traumatol Buco-Maxilo-Fac. 2016; 16: $19-24$

4. Nguyen DC, Shahzad F, Snyder-Warwick A, Patel KB, Woo AS. Transcaruncular Approach for treatment of medial wall and large orbital blowout fractures. Craniomaxillofac Trauma Reconstr. 2016; 9: 46-54.

5. Calderoni DR, Guidi MC, Kharmandayan P, Nunes PHF. Seven-year institutional experience in the surgical treatment of orbito-zygomatic fractures. J Craniomaxillofac Surg. 2011; 39: 593-9. doi: 10.1016/j.jcms.2010.11.018

6. Lock JZ, Hegde R, Young S, Lim TC, Amrith S, Sundar G. A study of sports-related orbital fractures in Singapore. Orbit. 2017; 36: 301-6.

7. Chou C, Kuo YR, Chen CC, Lai CS, Lin SD, Huang SH, et al. Medial orbital wall reconstruction with porous polyethylene by using a transconjunctival approach with a caruncular extension. Ann Plast Sur. 2017; 78(3 Suppl 2): 89-94.

8. Timóteo CA, Chagas JFS, Rapoport A, Denardin OVP. Avaliação da abordagem palpebral subtarsal no tratamento cirúrgico das fraturas zigomático-orbitais. Rev Col Bras Cir. 2009; 36: 382-91.

9. Kim YH, Park Y, Chung KJ. Considerations for the management of medial orbital wall blowout fracture. Arch Plast Surg. 2016; 43: 229-36.

10. El-Anwar MW, Elsheikh E, Hussein AM, Tantawy AA, Abdelbaki YM. Transconjunctival versus subciliary approach to the infraorbital margin for open reduction of zygomaticomaxillary complex fractures: a randomized feasibility study. Oral Maxillofac Surg. 2017; 21: 187-92. 